Volume 8. No. 7, July 2020

International Journal of Emerging Trends in Engineering Research

Available Online at http://www.warse.org/IJETER/static/pdf/file/ijeter137872020.pdf https://doi.org/10.30534/ijeter/2020/137872020

\title{
Disease Prediction and Performance Analysis using Data Analytics
}

\author{
Shruthi $\mathbf{J}^{1}$, Mamatha Bai B G ${ }^{2}$ \\ ${ }^{1}$ M. Tech Scholar, Computer Science \& Engineering, Nitte Meenakshi Institute of Technology, Bangalore, India, \\ shruthij1997@gmail.com \\ ${ }^{2}$ Assistant Professor, Computer Science \& Engineering, Nitte Meenakshi Institute of Technology, Bangalore, \\ India, mamathamane@gmail.com
}

\begin{abstract}
Big Data means the large and the voluminous data collected from various resources. The data helps us in understanding lot many aspects of the particular field. Healthcare is one such field or area which produces huge number of data such as patient's profiles, disease, symptoms and so on. Data Analytics comes into the picture when we have to review, analyze and predict the data. The main objective of the Data Mining Techniques is to make smart decisions, consume less time and being more accurate. In this paper, we have used two different datasets: Pregnancy Diabetes Dataset and Breast Cancer Dataset. The Classification Algorithms like SVM and Random Forest for the top-level classification and Clustering Algorithms like Agglomerative, Gaussian Mixture Model, and Spectral Clustering for further processing. The efficient algorithm is identified by comparing the accuracy results of the different implemented clustering algorithms.
\end{abstract}

Key words: Agglomerative, Big Data Analytics, Breast Cancer, Data Mining, GMM, Healthcare, Pregnancy Diabetes, Random Forest, Spectral, SVM,.

\section{INTRODUCTION}

Big Data is one of the main areas that needs more focus and more research activities are taking place. Big data is the combination and collection of structured, unstructured and semi-structured data [1]. In the current world full of technology and innovations, Big Data Analytics have achieved the at most importance and it is the developing technology leading for creating a range of curiosity with the advancements in the application areas. With innovation progression, the period of information in different technicalities, is developing liberally and taking care of those information may be a major summons in genuine constraint. It bargains with the exclusive degree of information and examination, which retrieves the result instantaneously. As we all know, it is a vast collection of information \& bewildered information that can be intense to bargain with ordinary actualities. Big Data rearranges the unorganized information to the productive experiences that is advantageous in keeping up the mind blowing sum of information received through different sources. This thought of managing the expansive sum of information is not modern but there could be a better approach in handling it. Healthcare in Big Data is one of the most important fields which have worked with the information that deals with the $5 \mathrm{~V}$ 's that's Velocity, Variety, Volume, Veracity and Value which are natural components of the realities. The truth is unfurling among a few of healthcare, composition, wellness, insurers, researcher's rights, organization etc. The characteristic complexity of the healthcare truths the advantage in creating and implementing big data caches and detects the arrangements to the issues that are being raised. Traditional forms of science have usually targeted the examination of diseases which are based on the modifications within the form of confined insights of a particular methodology of information.

\subsection{Importance of Big Data in Healthcare}

The role of Big Data in Healthcare is gaining importance corresponding to the volume of information which is either structured or unstructured. Medical data is confidential and ought to be taken care of. Analysis of the diseases based on the symptoms makes a difference where the specialists and patients induce to know about the wellbeing conditions at an early stage and accordingly follow preventive measures. Big data provides the strategy of standard information frameworks [2]. The data is too much colossal and dealing with the genuine truths can be a great challenge within the field of Healthcare. Dealing with huge data implies to electronic wellbeing and is troublesome to give away with ancient or essential data administration methods and old-fashioned bundle.

\subsection{Pregnancy Diabetes}

Gestational diabetes is diabetes analyzed during preliminary time of pregnancy. Like other types of diabetes, gestational diabetes influences how cells utilize glucose. Gestational diabetes causes high level blood sugar that can influence the pregnancy and the fetus.

Those who create gestational diabetes are at higher hazard of creating type- 2 diabetes in future. In most cases, there are no indications. A blood sugar test during pregnancy is 
diagnosed. Treatment procedures incorporate day by day blood sugar observing, a solid count calories, work out and checking the infant. On the off chance that blood sugar is as well high, pharmaceutical assistance is required.

The diabetes during pregnancy appears in 3 different stages: "Low", "Mid" and "High". For a few ladies, diabetes start and end during their pregnancy period. But for few, it is carried throughout their life. Some of the babies do get affected from the mother's womb itself.

\subsection{Breast Cancer}

Breast cancer gets created in breast cells. Regularly, the cancer shapes in either the lobules or the channels of the breast. Lobules are the organs that create drain, and channels are the pathways that bring the drain from the organs to the areola. Breast cancer can happen in ladies and seldom in men. Symptoms of breast cancer incorporate a protuberance within the breast, ridiculous release from the nipple and changes within the shape or surface of the areola or breast. Its treatment depends on the stage of cancer. It may comprise of chemotherapy, radiation, hormone treatment and surgery. The anatomy of the breast cancer tumor can be seen in the Figure 1.

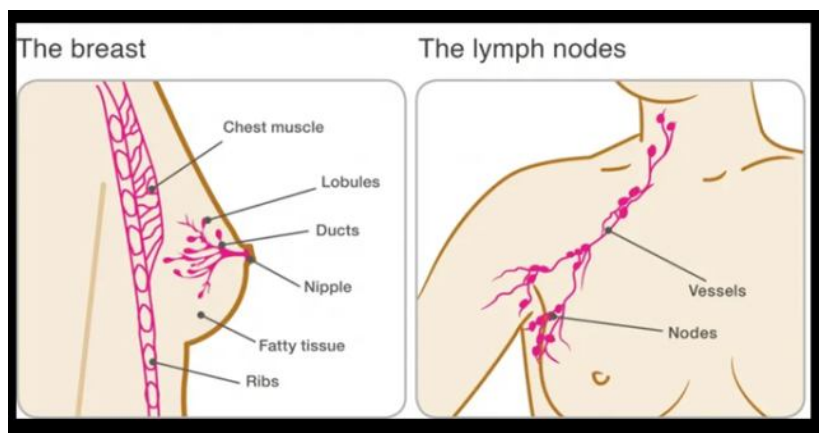

Figure 1: The anatomy of Breast Cancer and the Tumor

Breast Cancer occurs in 4 different stages, named such as "Pre-cancer", "Intermediate Cancer", "Advanced Cancer" and "Meta-static Cancer", which is shown in the Figure 2.

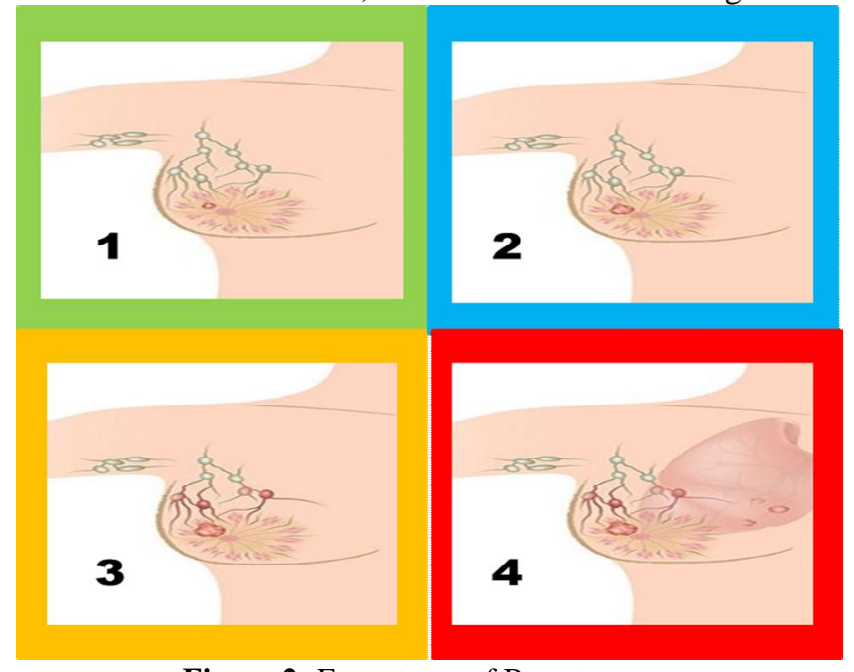

Figure 2: Four stages of Breast cancer

\section{LITERATURE SURVEY}

In [3] Data mining approaches offer the methodology and technology to transform these heterogeneous data into meaningful information for decision making. Large number of healthcare dataset is considered. Keeping $\mathrm{k}$ means as reference, $\mathrm{G}$ means algorithm was developed. Clustering methodologies are very efficient. Both algorithm and data taken should be pre-processed using specific rules so that they can enhance the performance and the results of the clustering algorithm. [4] GMM is the simplest algorithm which gives the efficient result and accuracy. GMM uses good number of parameters to get the result out of the trained data. [5] Random Forest has given the better accuracy. It has the advantage of computing the importance of each variable in the classification process. In the same way for our work we have used healthcare dataset, adopted Random Forest and SVM for the classification. AdaBoost, Random Forest was used as base classifier (93.3\%) [6].

In [7] Breast cancer is studied and spectral clustering is implemented. It is adopted in our work for detecting he stages of the disease. Spectral clustering can be achieved directly. [8] explains Constrained Spectral Clustering with the performance of a series of classic models in terms of classification accuracy with respect to whether individual's reported symptoms increase or decrease over time Health care has been taken as the work. In [9], the average accuracy of proposed model is $85.59 \%$ which is higher as compared to other models proposed earlier. . SVM classification algorithm is used to classify the data into different number of classes. The algorithms like EM, ANN, C4.5 have contributed in the analysis of data on kidney disease which referred as paper [10]. The algorithms have proven themselves on contribution by classifying the data into accurate classes and also providing the great accuracy of $70 \%, 75 \%$ and $96.5 \%$ respectively. Mamatha Bai B G et. al. [11] has focused on Gaussian Naïve Bayes, OPTICS and BIRCH algorithms for the analysis of Diabetes. Analysis of these algorithms is done by comparing the accuracy and by changing the various metrics of the performance. And concludes the OPTICS Algorithm gives the best result and is suitable for the given dataset.

The publication on the Data Mining Technology for Video Summarization can be seen in the paper [12] which makes use of CURE algorithm for both big data and video summarization streams. The algorithm gives the best results for both the streams. The paper [13] is aimed to study and compare the effect of bagging on classification accuracy by using different decision trees as the base classifiers. The experiment shows the effect of bagging on various base classifiers. The various statistical studies carried out for different algorithms for medical data is presented in [14]. 
Madam Chakradar et. al. [15] has explained about Support Vector Machine, Logistic Regression and other algorithms for Diabetes Mellitus of Type 2. The paper explains about the finding insulin through Machine Learning Algorithms. The accuracy of $97 \%$ is achieved. In [16], the author explains about the mobile application which will help the normal people to keep track on their blood pressure, using microcontrollers and also using sensors to detect the blood pressure.

\section{PROPOSED METHODOLOGY}

This work predicts the presence of Diabetes and Breast Cancer and performance analysis of various algorithms implemented. Figure 3 represents the work flow of the proposed method. The figure describes the whole architecture of the system, the flow of the algorithms from the data pre-processing to the results. The proposed methodology uses both classification and clustering technologies.

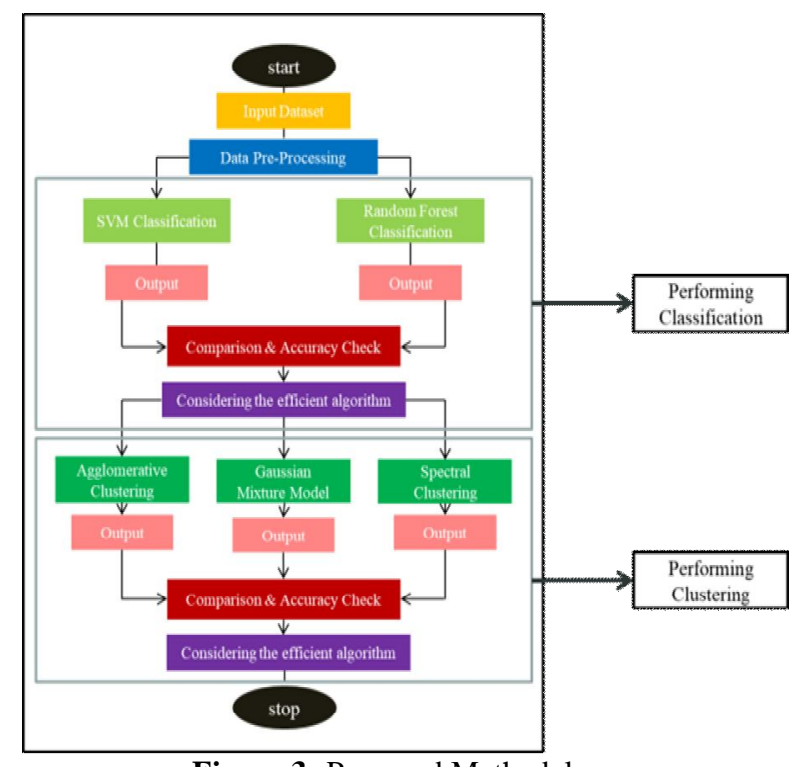

Figure 3: Proposed Methodology

The dataset is read in the first step and pre-processed for the missing values. Once it is done, the classification techniques are applied which further forms the classes based on the dataset for top level classification. Further the affected dataset is fed to the clustering algorithms for the deeper level clustering, where the levels and the stages of the disease is being predicted and further performance analysis is done.

\subsection{Dataset Description}

\section{A. Pregnancy Diabetes Dataset}

The dataset is taken from the source [17]. The dataset contains 1000 records of the patients with 9 varied attributes, which is listed in the Table 1.
Table 1: Attributes of Pregnancy Diabetes Dataset

\begin{tabular}{|c|c|}
\hline Sl. No. & Attributes \\
\hline 1. & Patient Id \\
\hline 2. & Plasma Glucose Level \\
\hline 3. & Diastolic Blood Pressure \\
\hline 4. & Triceps Thickness \\
\hline 5. & Serum Insulin \\
\hline 6. & Body Mass Index \\
\hline 7. & Diabetes Pedigree \\
\hline 8. & Age \\
\hline 9. & Class \\
\hline
\end{tabular}

\section{B. Breast Cancer Dataset}

The dataset is taken from the source [18]. The dataset contains 10,000 records of the patients with 10 varied attributes, which are calculated for the values listed in the Table 2.

Table 2: Attributes of Breast Cancer Dataset

\begin{tabular}{|c|c|}
\hline Sl. No. & Attributes \\
\hline 1. & Radius \\
\hline 2. & Texture \\
\hline 3. & Perimeter \\
\hline 4. & Area \\
\hline 5. & Smoothness \\
\hline 6. & Compactness \\
\hline 7. & Concavity \\
\hline 8. & Concave points \\
\hline 9. & Symmetry \\
\hline 10. & Fractal Dimensions \\
\hline
\end{tabular}

\subsection{Algorithms}

\section{A.Support Vector Machine (SVM)}

\section{Algorithm: SVM \\ Input: Dataset to classify whether Diseased or healthy \\ Output: Two Classes predicting the presence of Disease Steps:}

1. At first, what SVM does is to find the line which separates the two classes.

2. The taken dataset is fed to the algorithm

3. Finds out the data points that are the closest to the line from both the classes. Maximizing the margin is the major goal, which is called as the hyper plane.

4. $\mathbf{z}=\mathbf{x}^{2}+\mathbf{y}^{2}, \mathbf{z}$ coordinate is the squared distance of the data point from the origin. We add the $\mathrm{z}$ coordinate to make the data points linearly separable.

5. Thus we can keep adding the extra dimensionalities to make it linearly separable. 
Shruthi J et al., International Journal of Emerging Trends in Engineering Research, 8(7), July 2020, 3744 - 3752

\section{B. Random Forest Classification}

Algorithm: Random Forest

Input: Dataset to classify whether Diseased or healthy

Output: Two Classes predicting the presence of Disease

Steps:

1. Input the given dataset

2. Divide the dataset into training and testing data

3. Selection of samples randomly for the given datasets.

4. Decision tree is constructed for every sample by the algorithm; prediction results from each decision tree are retrieved.

5. Algorithm will perform voting for every predicted result.

6 . The most voted result is decided as the best solution for the dataset given.

\section{C.Agglomerative Clustering}

Algorithm: Agglomerative Clustering

Input: Dataset to predict the stages of the disease

Output: Three clusters predicting the stages of Disease

Steps:

1. The data set given will have (p1, p2, p3, .........,pn) of size $\mathrm{N}$

2. It computes the distance matrix

3. for $(\mathrm{i}=1$ to $\mathrm{N})$

4. for $(\mathrm{j}=1$ to $\mathrm{N})$

5. distance_matrix $[\mathrm{i}][\mathrm{j}]=$ dis $[\mathrm{p} 1, \mathrm{p} 2]$ here, each point is considered as individual clusters.

6. Now, repeat the steps for two clusters to merge into one, which has the closest distance.

7. Repeat until all the clusters are being merged.

\section{D.Gaussian Mixture Model (GMM)}

\section{Algorithm: GMM}

Input: Dataset to predict the stages of the disease

Output: Three clusters predicting the stages of Disease Steps: The Steps of the Gaussian Mixture Model can be seen from the Figure 4.

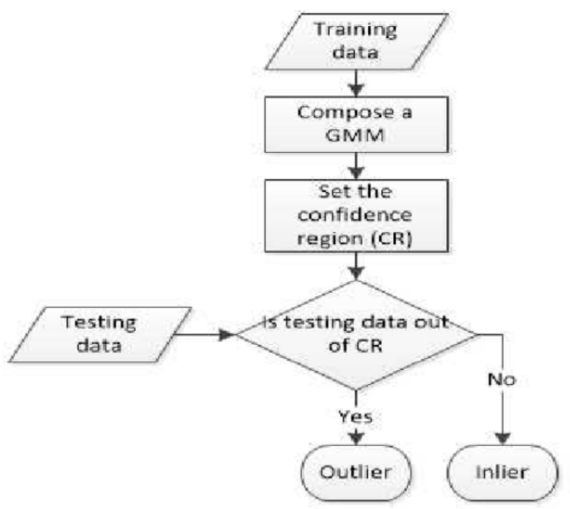

Figure 4: Representing the workflow of the GMM

\section{E. Spectral Clustering}

Algorithm: Spectral Clustering

Input: Dataset to predict the stages of the disease

Output: Three clusters predicting the stages of Disease

Steps:

1. Building similarity graph using:
a. Epsilon
b. K-Nearest Neighbors
c. Fully Connected

2. Predict the data onto a lower Dimensional Space

3. Clustering of the Data

\section{EXPERIMENTAL RESULTS AND ANALYSIS}

The dataset was fed to the classification algorithms, where the first level of classification is done by dividing the dataset to two classes. For the Diabetic dataset, the classes are: Diabetic and Non Diabetic. For the Breast cancer, we have Malignant and Benign as two different classes. The corresponding graphs are represented in this section.

\subsection{Pregnancy Diabetes Data Analysis}

Classification for top level categories for Pregnancy Diabetes Dataset are done using SVM and Random Forest which can be seen in Figures 5 and 6.

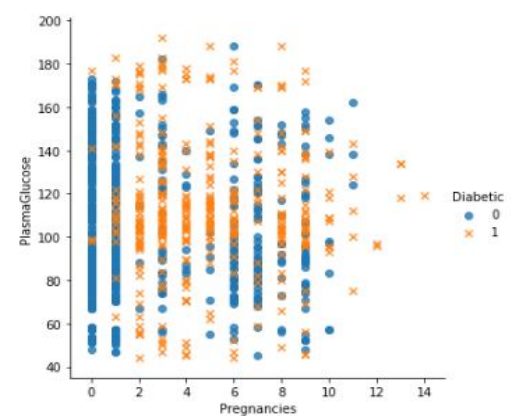

Figure 5: O/p of SVM classifier for Diabetic Dataset

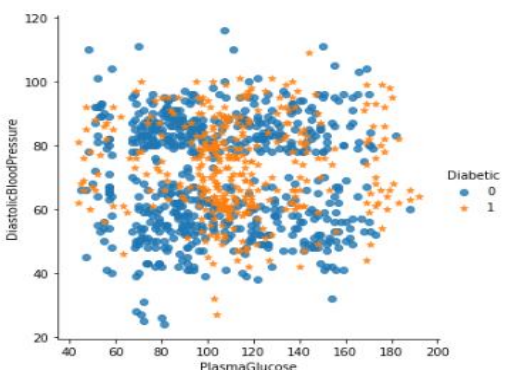

Figure 6: O/p of Random Forest classifier for Diabetic Dataset

Further, we proceed to find out the severity stages by using the clustering algorithms, where the clusters are formed using the clustering algorithms.

Clusters were formed based on the stages of Diabetes such as

- Low

- Mid

- High 


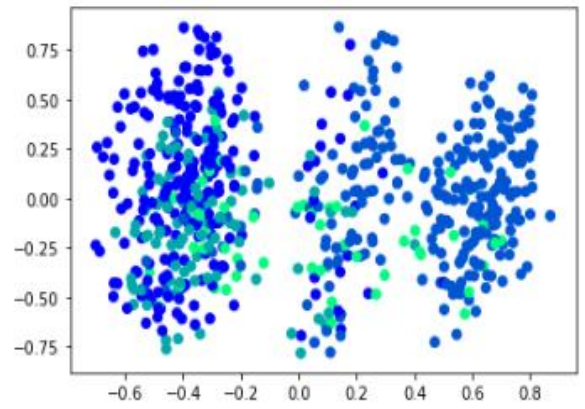

Figure 7: O/p of Agglomerative Clustering for Diabetic Data

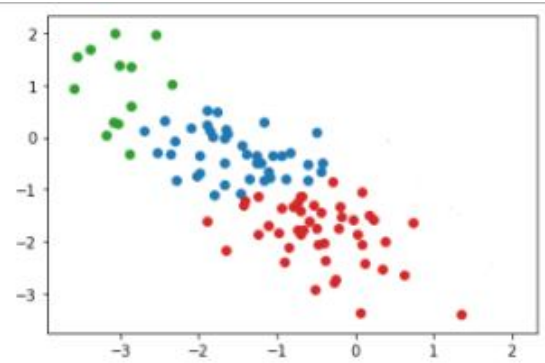

Figure 8: O/p of GMM for Diabetic Data

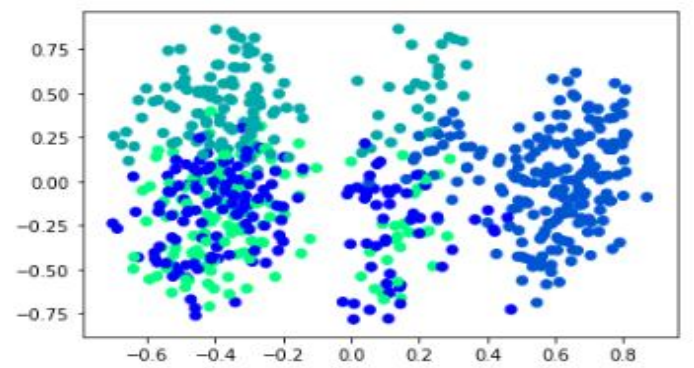

Figure 9: O/p of Spectral Clustering for Diabetic Data

Each color in the graphs denotes different clusters based on the stages. We can notice in Figures 7, 8 and 9 that there are three different clusters denoting the three different stages of the diabetes.

\subsection{Breast Cancer Data Analysis}

Classification for top level categories for Breast Cancer Dataset are done using SVM and Random Forest which can be seen in Figures 10 and 11.

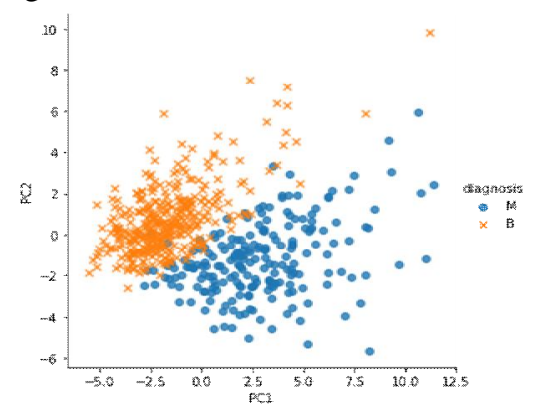

Figure 10: O/p of SVM classifier for Breast Cancer Data

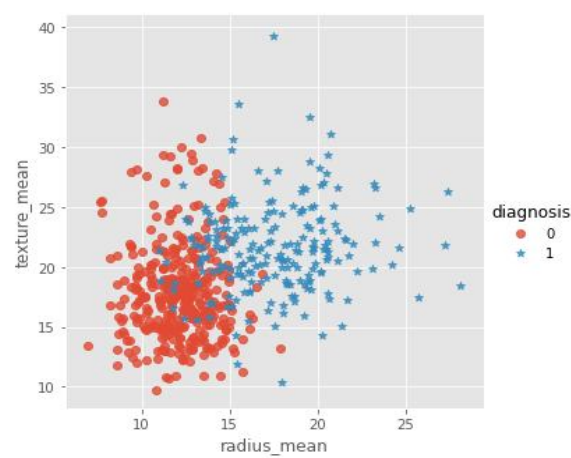

Figure 11: O/p of Random Forest classifier for Breast Cancer Data

Further, we proceed to find out the severity stages by using the clustering algorithms, where the clusters are formed using the clustering algorithms.

Clusters were formed based on the stages of breast cancer such as:

- Stage-1: Pre-Cancer

- Stage-2: Intermediate Cancer

- Stage-3: Advanced Cancer

- Stage-4: Meta-static Cancer

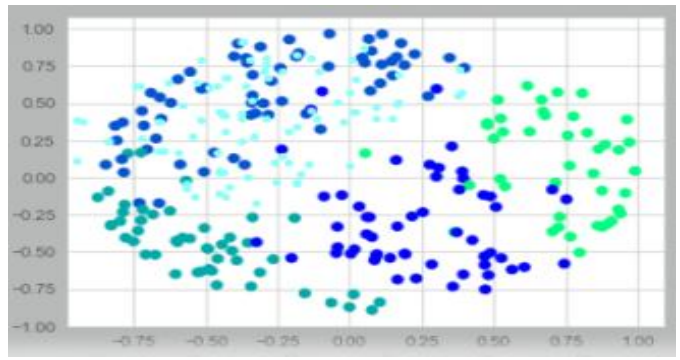

Figure 12: $\mathrm{O} / \mathrm{p}$ of Agglomerative for Breast Cancer Data

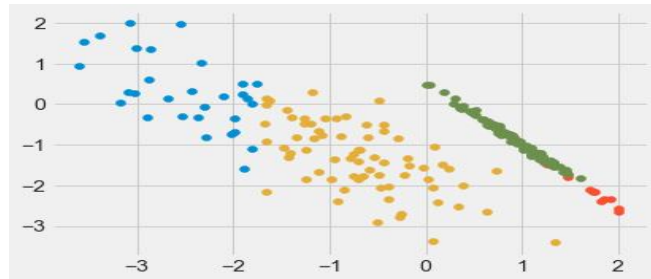

Figure 13: O/p of GMM for Breast Cancer Data

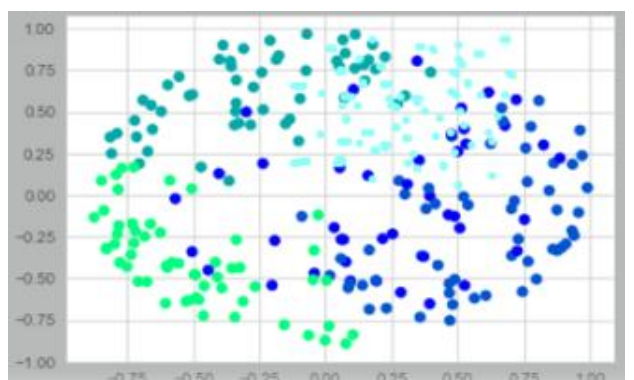

Figure 14: $\mathrm{O} / \mathrm{p}$ of Spectral clustering for Breast Cancer Data

Each color in the graphs denotes different clusters based on the stages. We can notice in Figures 12, 13 \& 14 that there are three different clusters denoting the three different stages of the diabetes. 


\section{STATISTICAL ANALYSIS}

Table 3 represents the accuracy of all the algorithms that are placed against each other for the better understanding.

We can observe that from the classification algorithms, SVM gives the better results for both the datasets, and in the clustering algorithms GMM method gives us the better accuracy.

Table 3: Accuracy of Algorithms

\begin{tabular}{|l|c|c|}
\hline \multicolumn{1}{|c|}{ Algorithm } & Diabetes & Breast Cancer \\
\hline SVM & $96.4 \%$ & $97.2 \%$ \\
\hline Random Forest & $96.1 \%$ & $96.5 \%$ \\
\hline GMM & $81 \%$ & $79 \%$ \\
\hline Agglomerative & $67 \%$ & $65.6 \%$ \\
\hline Spectral & $61 \%$ & $62.3 \%$ \\
\hline
\end{tabular}

\subsection{Statistical analysis of the pregnancy diabetes dataset.}

Figure 15 \& 16 represent the classification algorithms results. The percentage representations of the division of categories are depicted using the graphical visualization.

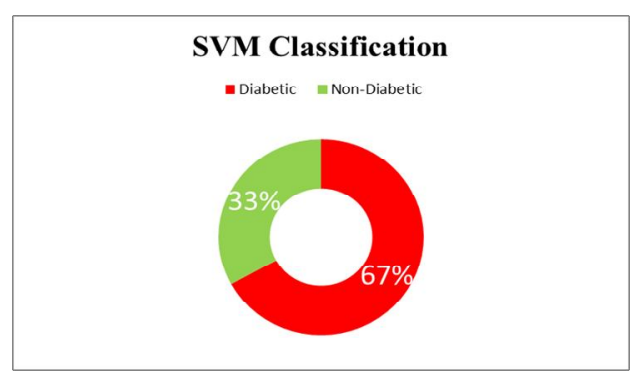

Figure 15: Graphical representation of the prediction results obtained by SVM for Diabetic Data

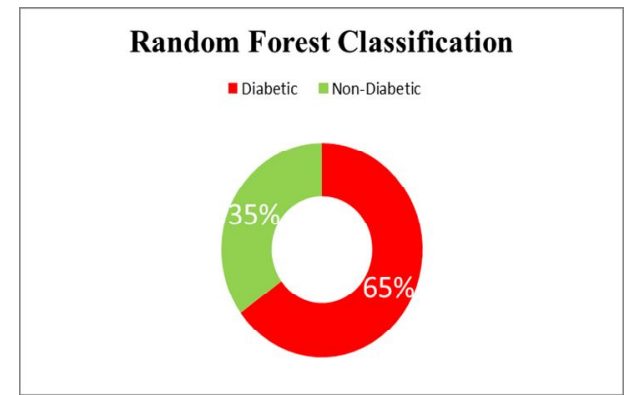

Figure 16: Graphical representation of the prediction results obtained by Random forest for Diabetic Data.

The prediction results obtained by different Clustering Algorithms for Diabetic Data is graphically represented in Figure 17.

\subsection{Statistical analysis of the breast cancer dataset.}

Figures 18 \& 19 represent the classification algorithms results. The percentage representations of the division of categories are being depicted using the graphical visualization.

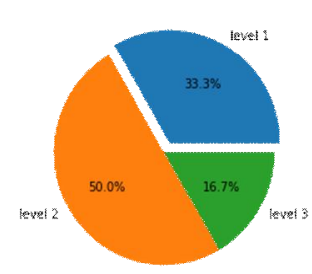

Figure 17(a): Agglomerative Clustering

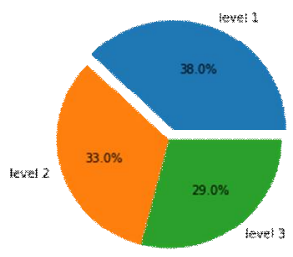

Figure 17(b): GMM

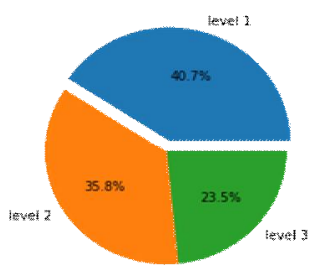

Figure 17(c): Spectral Clustering

Figure 17: Graphical Representation of Prediction results obtained by different Clustering Algorithms for Diabetic Data

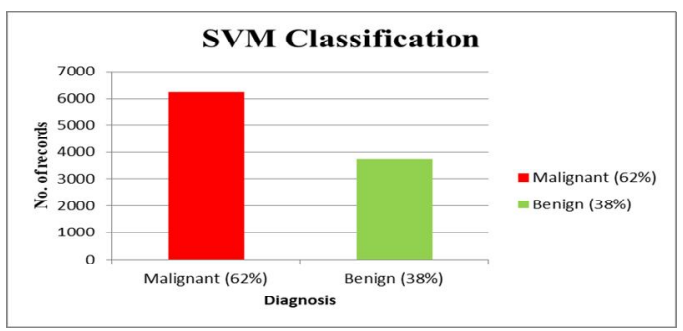

Figure 18: Graphical representation of the prediction results by SVM for Breast Cancer

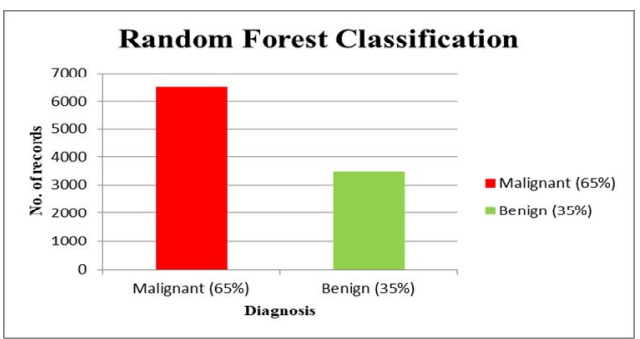

Figure 19: Graphical Representation of the prediction results by Random Forest for Breast Cancer

The statistical analysis for Breast Cancer using clustering algorithms is illustrated in Figures 20, 21 and 22. 
Shruthi J et al., International Journal of Emerging Trends in Engineering Research, 8(7), July 2020, 3744 - 3752

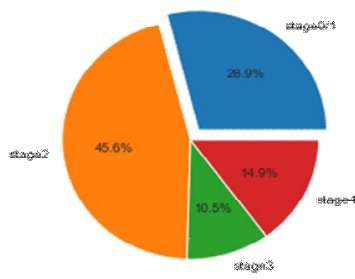

Figure 20: Graphical representation of the prediction results obtained by Agglomerative Clustering for Breast Cancer

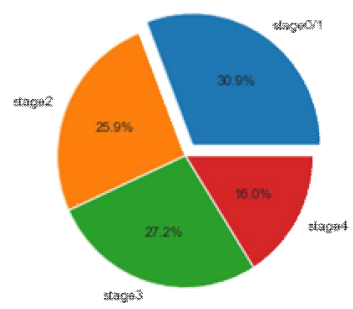

Fig 21: Graphical representation of the prediction results obtained by GMM for Breast Cancer

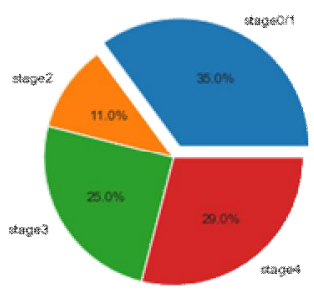

Figure 22: Graphical representation of the prediction results obtained by Spectral Clustering for Breast Cancer

\section{WEB APPLICATION RESULTS}

The user interface is the one that we can experience and use it as platform for sharing information. Having this thought, the results obtained from the algorithms, their accuracies, statistical results are constituted using HTML and CSS technology, where one can use the website to refer the results and analyze further.

Figure 23 shows the Welcome Page of the Website developed followed by the Information Page in Figure 24. Classification and Clustering Results in the UI environment are shown in Figures 25 and 26 respectively. Figure 27 and 28 depicts the Accuracy and Statistical analysis of our work.

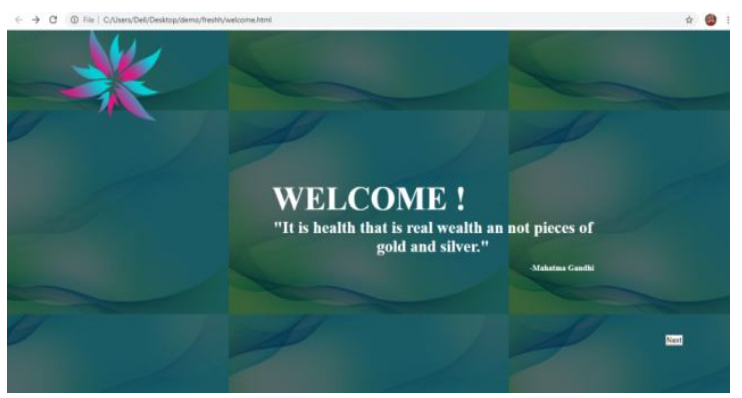

Figure 23: Welcome Page

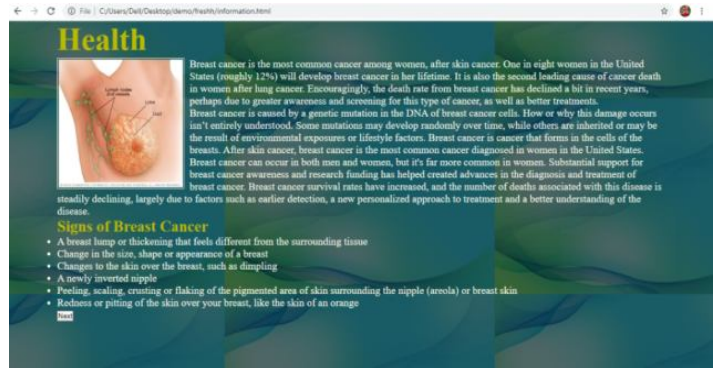

Figure 24: Information Page

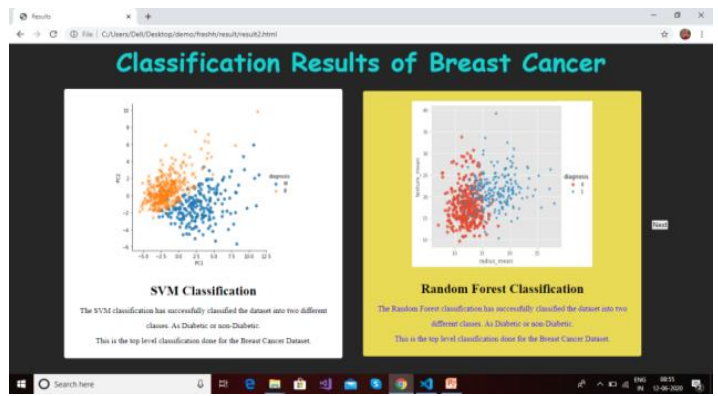

Figure 25: Classification Results in the UI

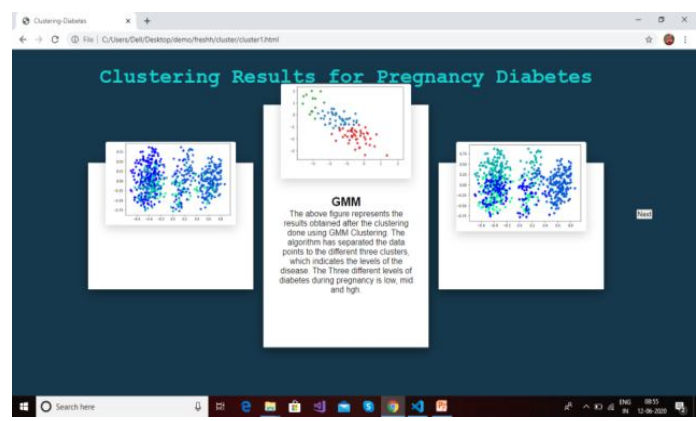

Figure 26: Clustering Results in the UI

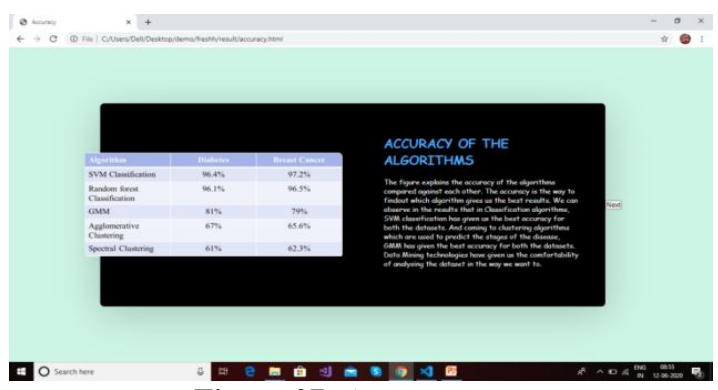

Figure 27: Accuracy page

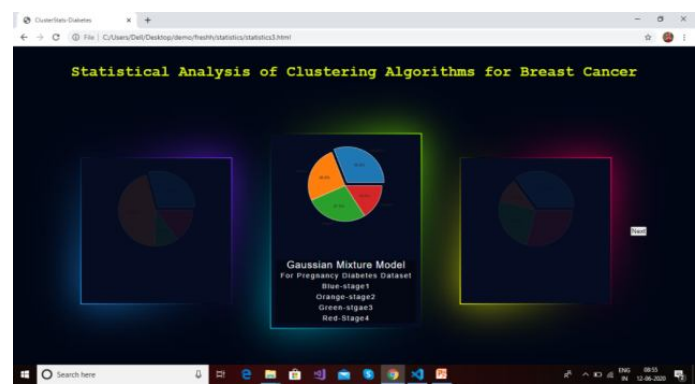

Figure 28: Statistical Analysis page 


\section{CONCLUSION AND WAY FORWARD}

The accuracy of the each algorithm is being compared against each other. We can observe that SVM classifier under classification algorithms for top level classification gives the highest accuracy for the datasets used. And the GMM model under clustering algorithms, gives the highest accuracy for both the datasets.

The main objective and motivation of this work is all about the women's healthcare, be it a diabetes or breast cancer and to create awareness among the Women Community especially in the rural sectors. Further, we can develop an android application which will be an Interactive platform for the users to know about the disease and also to create the public awareness.

\section{ACKNOWLEDGEMENT}

The authors express their sincere gratitude to Prof. N R Shetty, Advisor and Dr. H C Nagaraj, Principal, Nitte Meenakshi Institute of Technology for giving constant support to carry out research at NMIT.

\section{REFERENCES}

1. Scardapane, Rosa Altilio, Valentina Ciccarelli, Aurelio Uncini and Massimo Panella, "Privacy-Preserving Data Mining for Distributed Medical Scenarios", 00184 Rome, Italy of the Springer International Publishing AG 2018. https://doi.org/10.1007/978-3-319-56904-8_12

2. B G Mamatha Bai, R Harshitha, Jharna Majumdar, "Diagnosis of Cardiotocography for Analysis and Prediction of Treatment during Pregnancy", Proceedings of 2018 International Conference on Advances in Computing, Communications and Informatics, IEEE Explore, ISBN: 978-1-5386-5314-2, DOI: 10.1109/ICACCI.2018.8554437.

3. Ramzi.Haraty, MohamadDimishkieh, and MehediMasud "An Enhanced k-Means Clustering Algorithm for Pattern Discovery in Healthcare Data" Proceedings of the Industrial and Systems Engineering Research Conference, 2018.

4. Felicity $R$ Allen, Eliathamby Ambikairajah, Nigel $H$ Lovell, and Branko G Celler, National Information and Communications Technology Australia, "Classification of a known sequence of motions and postures from accelerometry data using adapted Gaussian mixture models" Received 12 February 2016, accepted for publication 27 June 2016, Published 25 July 2017.

5. Khalilia, Sounak Chakraborty and Mihail Popescu, "Predicting disease risks from highly imbalanced data using random forest" USAKhalilia et al. BMC Medical Informatics and Decision Making 2018.

6. María N. Moreno García, Juan Carlos Ballesteros Herráez, Mercedes Sánchez Barba and Fernando Sánchez Hernández, "Random Forest Based Ensemble
Classifiers for Predicting Healthcare-Associated Infections in Intensive Care Units" 13th International Conference, Advances in Intelligent Systems and Computing 474, 2016

7.B. Walker, Jacob N. Norris, Anna E. Tshiffely, Melissa L. Mehalick, Craig A. Cunningham, Ian N. Davidson, Senior Member, "Applications of Transductive Spectral Clustering Methods in a Military Medical Concussion Database" IEEE/ACM Transactions on Computational Biology and Bioinformatics Proceedings of the 2016 IEEE TRANSACTIONS ON COMPUTATIONAL BIOLOGY AND BIOINFORMATICS.

https://doi.org/10.1109/TCBB.2016.2591549

8. Chua Kuang Chua, Vinod Chandran, Rajendra U. Acharya and Lim Choo Min 1Department of Electronics and Computer Engineering "Cardiac Health Diagnosis Using Higher Order Spectra and Support Vector Machine", The Open Medical Informatics Journal, 2016, 3, 1-8.

9. Divya Tomar and Sonali Agarwal Indian Institute of Information and Technology, "Feature Selection based Least Square Twin Support Vector Machine for Diagnosis of Heart Disease" Journal of Bio-Science and Bio-Technology Vol.6, No.2 (2017).

https://doi.org/10.14257/ijbsbt.2014.6.2.07

10. Tabassum S, Mamatha Bai B G, Dr. Jharna Majumdar, "Analysis and Prediction of Chronic Kidney Disease using Data Mining Techniques", International Journal of Engineering Research in Computer Science and Engineering (IJERCSE) ISSN (Online) 2394-2320, Vol 4, Issue 9, September 2017, DOI: 10.13140/RG.2.2.26856.72965.

11. Mamatha Bai B G, Nalini B M, Jharna Majumdar, "Analysis and Detection of Diabetes using Data Mining Techniques - A Big Data Application in Healthcare", Proceedings of Emerging Research in Computing, Information, Communication and Applications, Vol 1, Advances in Intelligent Systems and Computing, ISBN 978-981-13-5952-1 ISBN 978-981-13-5953-8 (eBook), DOI: org/10.1007/978-981-13-5953-8.

12. Jharna Majumdar, Mamatha Bai B G, Sumant Udandakar, "Implementation of CURE Clustering Algorithm for Video Summarization and Healthcare applications in Big Data", Proceedings of Emerging Research in Computing, Information, Communication and Applications, Vol 2, ISBN: 978-981-13-6001-5, DOI: 10.1007/978-981-13-6001-5, Springer.

13. Debjani Panda, Ratula Ray, Azian Azamimi Abdullah, Satya Ranjan Dash "Popular Ensemble Methods: An Empirical Study" Journal of Artificial Intelligence Research, 2017.

14. Shylashree G, Mamatha Bai B G, Jharna Majumdar, "Statistical Analysis and Prediction of Medical Data using Data Mining Techniques ensuring Data 
Shruthi J et al., International Journal of Emerging Trends in Engineering Research, 8(7), July 2020, 3744 - 3752

Confidentiality", TOUCAN Research and Development, ISBN No.: 978-81-942952-8-0, 2019.

15. Madam Chakradar, Alok Aggarwal "A Machine Learning Based Approach for the Identification of Insulin Resistance with Non-Invasive Parameters using Homa-IR", International Journal of Emerging Trends in Engineering Research, 8(5), May 2020, 2055 2064

https://doi.org/10.30534/ijeter/2020/95852020

16. Edward B. Panganiban "Microcontroller-based Wearable Blood Pressure Monitoring Device with GPS and SMS Feature through Mobile App", International Journal of Emerging Trends in Engineering Research, 7(6), June 2019, 32-35 https://doi.org/10.30534/ijeter/2019/02762019

17. https://www.kaggle.com/uciml/pima-indians-diabetes-d atabase

18. https://www.kaggle.com/uciml/breast-cancer-wisconsindata 